\title{
Lo que Saben y Piensan Enseñar los Futuros Profesores de Escuela Primaria sobre el Concepto de Materia. Estudio de la Amplitud, Diversidad y Organización Conceptual
}

Saúl A. Contreras, María A. Cruz, Alexis M. González

Departamento de Educación, Facultad de Humanidades, Universidad de Santiago de Chile, Avenida Libertador Bernardo O’Higgings 3363, Santiago-Chile. (email: saul.contreras@usach.cl)

Recibido Dic. 04, 2012; Aceptado Ene. 08, 2013; Versión final recibida Mar. 01, 2012

\section{Resumen}

Se ha investigado sobre el conocimiento disciplinar de 66 futuros profesores de primaria chilenos en relación a la amplitud, la diversidad y organización conceptual para la enseñanza del concepto de "materia". Para ello se utiliza un cuestionario abierto y un análisis cualitativo a través de mapas de organización y distribución conceptual. Los principales resultados indican que al momento de pensar en la enseñanza del tema "materia", los futuros profesores presentan un bajo conocimiento disciplinar. Este bajo conocimiento se caracteriza por una reducida diversidad, escasas relaciones conceptuales, dificultades para establecer conceptos de nivel microscópico y una ausencia total de concepto de nivel simbólico. Esto se resume en que ellos poseen un campo conceptual reducido.

Palabras clave: conocimiento disciplinar, concepto de materia, niveles de representación, organización conceptual

\section{What Primary School Teacher Trainees Know and Plan to Teach on the Concept of Matter. Study of the Amplitude, Diversity and Conceptual Organization}

\begin{abstract}
The disciplinary knowledge of 66 primary school trainee teachers has been investigated in relation to range, diversity, and conceptual organization for the teaching of the concept of "matter". An open-ended questionnaire was employed and a qualitative analysis of the data through maps of organization and conceptual distribution. The main results indicate that when the prospective teachers are questioned about the concept "matter" they a meager disciplinary knowledge. This poor knowledge is characterized by insufficient diversity, few conceptual relationships, difficulties in establishing microscopic level concepts and total absence of symbolic level concept. In other words they possess a reduced conceptual field.
\end{abstract}

Keywords: disciplinary knowledge, concept of matter, representation levels, conceptual organization 


\section{INTRODUCCIÓN}

Una parte importante del conocimiento profesional del profesor, lo constituye el conocimiento de los contenidos a enseñar, incluso antes de pensar en cómo enseñarlos. Shulman (2005) señala que el conocimiento didáctico (qué, cómo y cuándo enseñar) sobre un contenido en particular y que se desarrolla en la mente del profesor, se nutre del conocimiento disciplinar, conocimiento curricular y conocimiento pedagógico general que el profesor posea (Garritz y Trinidad-Velasco, 2004). Así, el conocimiento de la disciplina es un aspecto esencial para diseñar secuencias didácticas y enseñar, siendo determinante de la práctica del profesor en todos sus aspectos (Martín del Pozo y Rivero, 2001).

El conocimiento disciplinar se refiere a la organización y relación entre los conceptos del tema o tópico per se en la mente del profesor, por lo tanto, es primordial para decidir qué enseñar (Arteaga e Inciarte, 2008; Garritz y Trinidad-Velasco, 2004; Porlán, Rivero y Martín del Pozo, 1998; Tardif, 2004). Parece trivial pensar que para enseñar un contenido, primero es necesario conocer y comprender la organización conceptual que éste presenta, no obstante para ello se debe disponer de un conocimiento acabado sobre los hechos o conceptos de un determinado tema, se debe reconocer las estructuras de cómo se interrelacionan cada uno de los conceptos que incluyen dicho tema. Es decir, se debe reconocer la estructura sustantiva y sintáctica del contenido (Tardif, 2004). De esta manera, para que un profesor pueda enseñar un tema y logre transformar el conocimiento científico en un conocimiento escolar enseñable y aprendible, es necesario que maneje la fuente y naturaleza de la disciplina que enseña (Chevallard, 1991; Reyes y Garritz, 2006; Shulman, 2005). Un profesor con bajo conocimiento disciplinar, es menos consciente de las ideas previas de sus estudiantes y menos competente para identificar las dificultades conceptuales de los alumnos, proponer y organizar secuencias de contenidos y tiende a desarrollar prácticas de enseñanza basadas en la memorización (Carrillo et al., 2009).

Diversas investigaciones muestran que tanto profesores como futuros profesores, seleccionan y organizan los contenidos siguiendo una lógica disciplinar, aunque en muchos casos puedan tener dudas si ésta es la que permite lograr que los estudiantes aprendan (Sánchez y Valcárcel, 1999). No seleccionar y organizar de manera adecuada los contenidos disciplinares de un tema a enseñar, se relaciona, con prácticas tradicionales y con dificultades en los estudiante para aprender la estructura de los contenidos disciplinares e integrar sus ideas previas (Contreras, 2008; 2009; Martín del Pozo, 2001; Martínez y Espinoza, 2009). Por tanto, es fundamental un manejo disciplinar, por ejemplo de la química, pero también es fundamental un conocimiento y dominio de los niveles de representación de una disciplina, sobre todo para aquellas de naturaleza más abstracta. Solo a través de ello se puede comprender la estructura de un contenido, para luego enseñarlo (Casado y Raviolo, 2005; Galagovsky, 2005; 2007; Van Berkel et al., 2009).

La preocupación por desarrollar estándares disciplinares cobra sentido, si consideramos la necesidad de que los futuros profesores deben disponer de un conocimiento disciplinar que detalle los conceptos estructurantes de la disciplina que enseñarán. En este sentido, y para la enseñanza de la química, se señala que es necesario que los futuros profesores no solo posean un amplio conocimiento de los conceptos estructurantes (amplitud y diversidad), sino que además posean un dominio de las relaciones entre los conceptos (organización) y un dominio de los niveles de representación para su enseñanza (macroscópico, microscópico y simbólico), de manera que al momento de enseñar un tema, por ejemplo, la "materia", puedan establecer un eje vertebrador, representar y diseñar secuencias didácticas para mejorar los aprendizajes de sus estudiantes (Galagovsky, 2007; Gómez et al., 2004; Harrison y Treagust, 2003; Hilton y Nichols, 2011; Martín del Pozo, 2001; Zaid et al., 2012).

En este ámbito, pretendemos identificar y describir el conocimiento disciplinar de un grupo de futuros profesores chilenos de enseñanza primaria (general básica), centrándonos en la amplitud, diversidad y organización conceptual que poseen sobre el tema "materia", y los niveles de representación macroscópico, microscópico y simbólico utilizados en la explicitación de este conocimiento. Y así, responder a ¿Qué saben y piensan enseñar los futuros profesores de primaria sobre el tema la "materia"?

\section{METODOLOGIA}

En el marco de un proyecto DICYT (03-0854CP), esta investigación se desarrolló en dos fases (cualitativa y cuantitativa). Lo que se presentamos, es parte de la primera fase. La muestra está constituida por 66 futuros profesores de la Universidad de Santiago de Chile. Todos ellos, según año de ingreso $(2007,2008,2009)$ han recibido formación generalista, además de una formación en el área de ciencias (Estudio y Comprensión de la Naturaleza I, II y III). Además, todos provienen de instituciones de educación secundaria (media) pública y una mayoría son mujeres (62\%). El conjunto de futuros profesores fue dividido en tres grupos $\left(G_{1}, G_{2}\right.$ y $\left.G_{3}\right)$ según año de ingreso a la carrera $(2007,2008,2009)$ y se aplicó un cuestionario abierto, cuyas preguntas fueron: a) ¿Qué enseñarías del tema la "materia"? y b) ¿En qué orden? 
Teniendo en cuenta que se trabaja con una muestra reducida, el tratamiento de los datos que se obtienen de la producción escrita de los futuros profesores permite un análisis cualitativo de su contenido. Las producciones escritas, tanto de individuos como de grupos, fueron analizadas por categorías. Más específicamente, las categorías de análisis fueron: amplitud (numero), diversidad (tipos) y organización conceptual. Para esta última, se consideran relaciones horizontales entre conceptos (tramas conceptuales o conceptos estructurantes) y relaciones verticales (jerarquías conceptuales) (Martín del Pozo, 2001; Martínez y Espinoza, 2009; Moreira, 1998). De esta forma se utilizaron mapas de distribución y organización conceptual $y$, tablas de frecuencias.

Dado que pretendemos dar cuenta del inventario de conceptos implicados y los posibles aspectos del campo conceptual de un tema (Martín del Pozo, 2001), señalar que consideramos el conjunto de conocimientos, conceptos, sistemas conceptuales e información, para explicar en este caso el tema (contenido o tópico) "materia" (Mineduc, 2002; Garritz y Trinidad-Velasco, 2004). Así, para interpretar y explicar mejor los resultados, según nuestras categorías de análisis, distinguimos para el tema "materia", aspectos generales, específicos, cualitativos, cuantitativos y otros. Además, en los aspectos específicos distinguimos niveles (conceptos) macroscópico, microscópico y simbólico (Gilbert y Treagust, 2009; Martín del Pozo, 2001; Treagust y Chittleborough, 2004).

\section{RESULTADOS}

Los resultados se presentan según las tres categorías establecidas: diversidad, amplitud y organización. Para la primera, se utiliza un mapa de distribución conceptual (Fig. 1), para la segunda un gráfico de distribución (Fig. 2) y para la tercera, mapas conceptuales (Fig. 3, 4 y 5). Además, se describe un análisis comparativo $\left(\mathrm{G}_{1}, \mathrm{G}_{2}\right.$ y $\left.\mathrm{G}_{3}\right)$.

\section{Diversidad Conceptual}

Del conjunto de conceptos propuestos por los futuros profesores del grupo $G_{1}(N=16)$, no se observan conceptos para los aspectos cuantitativos, como tampoco para otros aspectos. La cantidad de conceptos propuestos es reducida y no se encuentran conceptos cuantitativos como por ejemplo volumen, peso y/o masa. Además, proponen el concepto cambio de estado (general) pero solo el de solidificación como específico. La misma tendencia se observa con el nivel microscópico, señalan átomo, pero no conceptos como núcleo atómico o electrones.

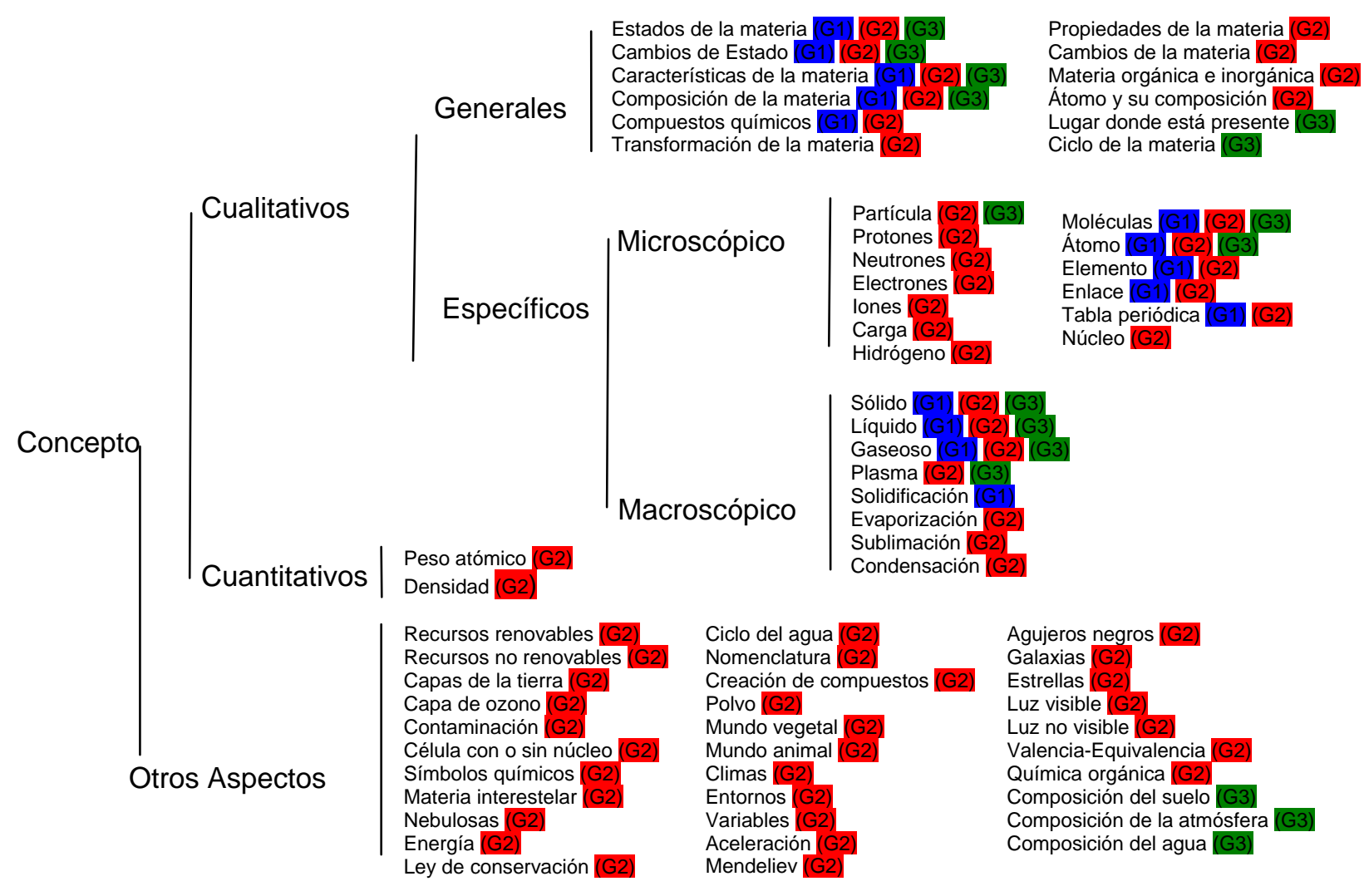

Fig. 1: Mapa de distribución conceptual (diversidad) 
El grupo $\mathrm{G}_{2}(\mathrm{~N}=20)$ es el único grupo que propone aspectos cuantitativos (densidad y peso atómico). Proponen además, conceptos que no tienen relación con el campo conceptual del tema "materia", agrupados en otros aspectos (recursos renovables, capas de la tierra, capa de ozono, célula, galaxias, etc.). En esta línea, proponen los conceptos específicos de sólido, líquido, gas y plasma para relacionarlos con el concepto general de estados de la "materia" y, solidificación, condensación, evaporación y sublimación con cambios de estado, faltando el de fusión. En relación a las propiedades de la "materia" no se observan aspectos específicos, como por ejemplo: compresibilidad, dureza, fluidez, volumen, masa, entre otros. Por otro lado, sobre los aspectos cuantitativos, aunque se observan dos conceptos asociados (densidad y peso atómico), es importante destacar que la relación de estos con el tema "materia" si bien no es incorrecta es preferible tratar en primera instancia los conceptos de volumen, peso y masa y, luego densidad.

El grupo $\mathrm{G}_{3}(\mathrm{~N}=30)$ propone conceptos para la mayoría de los aspectos, pero al igual que $\mathrm{G}_{1}$ no proponen aspectos cuantitativos. De los aspectos generales agrupados, se puede decir que el correspondiente a estados de la "materia" se relaciona con conceptos específicos como sólido, líquido, gas y plasma, y los que corresponden a características de la "materia" y composición de la "materia" con los conceptos específicos de molécula, átomo y partícula. En este sentido, los futuros profesores señalan conceptos relativos al contenido la "materia" más a nivel general, dado que la diversidad de conceptos relacionados con aspectos específicos es bastante reducida.

\section{Amplitud Conceptual}

$\left(\mathrm{G}_{1}\right)$ : del total de conceptos propuestos, una mayoría son macroscópicos. Específicamente, sólido, líquido, gas y composición de la "materia" (7). Por otro lado, aunque señalan dos conceptos microscópicos (átomo y enlace), sus frecuencias son muy bajas. Es decir, los conceptos microscópicos prácticamente están ausentes (1).

$\left(\mathrm{G}_{2}\right)$ : del total de conceptos propuestos, una mayoría son microscópicos. Ello podría suponer una visión más microscópica, al momento de pensar enseñar el tema "materia", sin embargo, al considerar la frecuencia con que es propuesto cada concepto, esta visión cambia. Por ejemplo, iones, carga, núcleo e hidrógeno presentan una frecuencia muy baja (1), mientras que los conceptos macroscópicos (sólido, líquido, gas, características y estados de la "materia") presentan una alta frecuencia (19).

$\left(G_{3}\right)$ : del total de conceptos propuestos, aquellos macroscópicos son los que presentan frecuencias más altas. Por ejemplo, estado de la "materia" (19), características de la "materia" (7), composición de la "materia" (5). Por otro lado, los aspectos microscópicos presentan menores frecuencias, entre ellos, partícula (6), átomo y molécula (3). Cabe señalar que los conceptos de sólido, líquido y gaseoso presentan una alta frecuencia (17), no obstante el concepto de plasma es propuesto solo 2 veces.

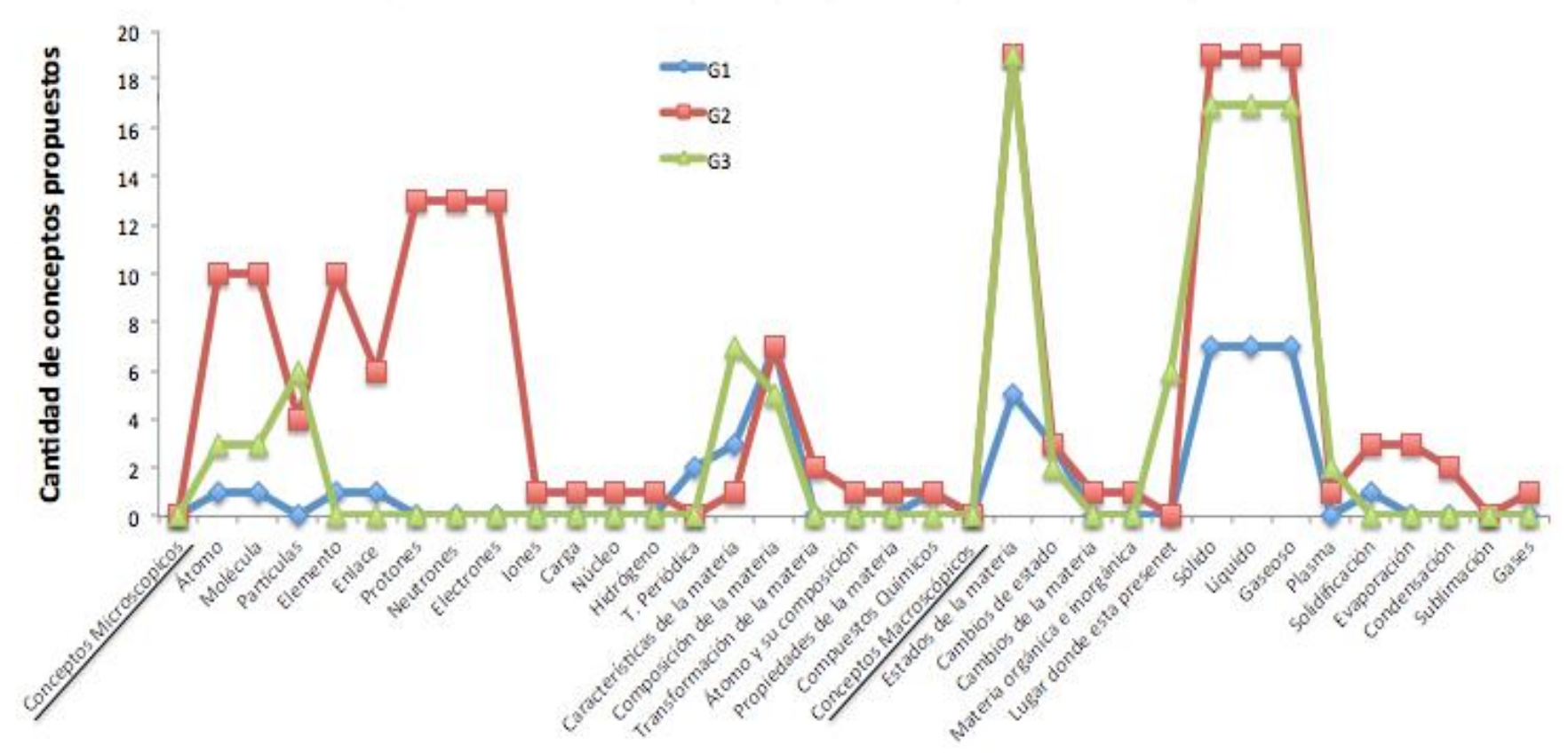

Fig. 2: Amplitud Conceptual 


\section{Organización Conceptual}

$\left(G_{1}\right)$ : el mapa de organización conceptual indica una relación vertical de los conceptos (Figura 3). Se observa una estructura de jerarquización que secuencia de lo general a lo particular. Una de las tres tramas no asocia conceptos, y las otras dos presentan relaciones inclusivas pero reducidas. Además, se confunde composición de la "materia" y características de la "materia".

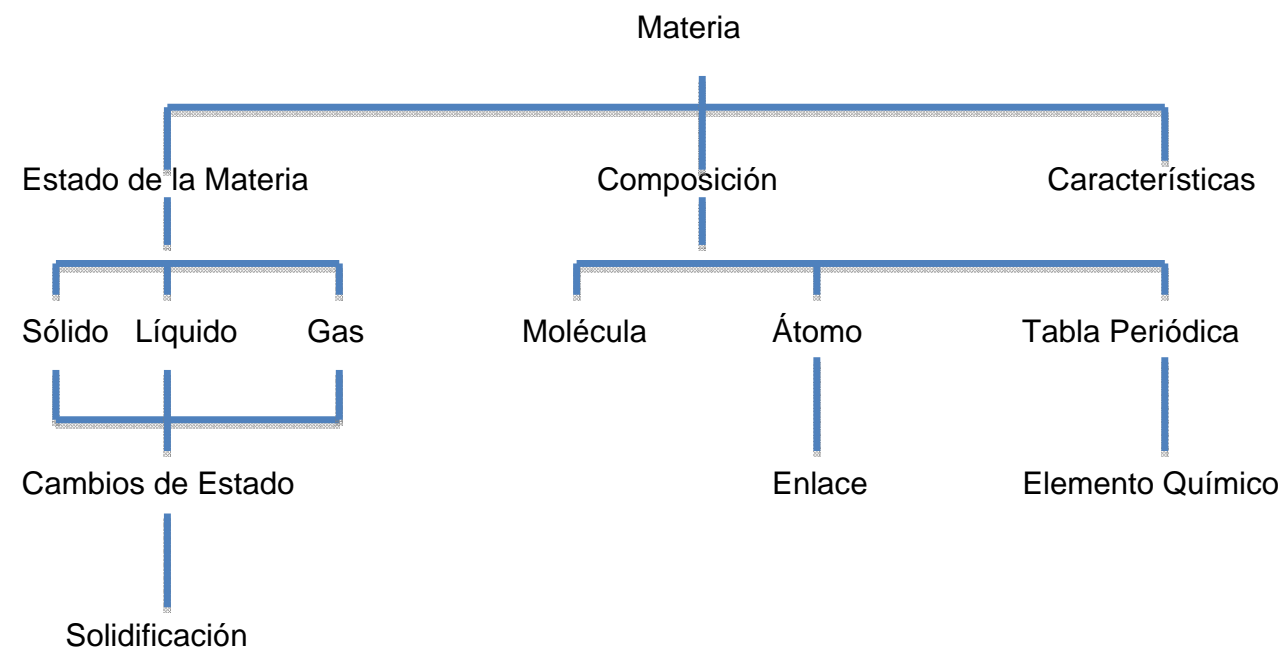

Fig. 3: Mapa de Distribución de Conceptos para $\mathrm{G}_{1}$

$\left(\mathrm{G}_{2}\right)$ : el mapa de organización conceptual presenta 27 conceptos. Se organizan cinco tramas y siete niveles jerárquicos (Figura 4). Las tramas correspondientes a transformaciones y características no presentan conceptos específicos asociados, el resto agrupa conceptos que tienen el mismo alcance dentro de la estructura de cada uno de los cuerpos de conocimientos. En los conceptos incluidos en las tramas, se observa una relación correcta entre los conceptos específicos y generales. Continúa confusión entre composición y características de la "materia".

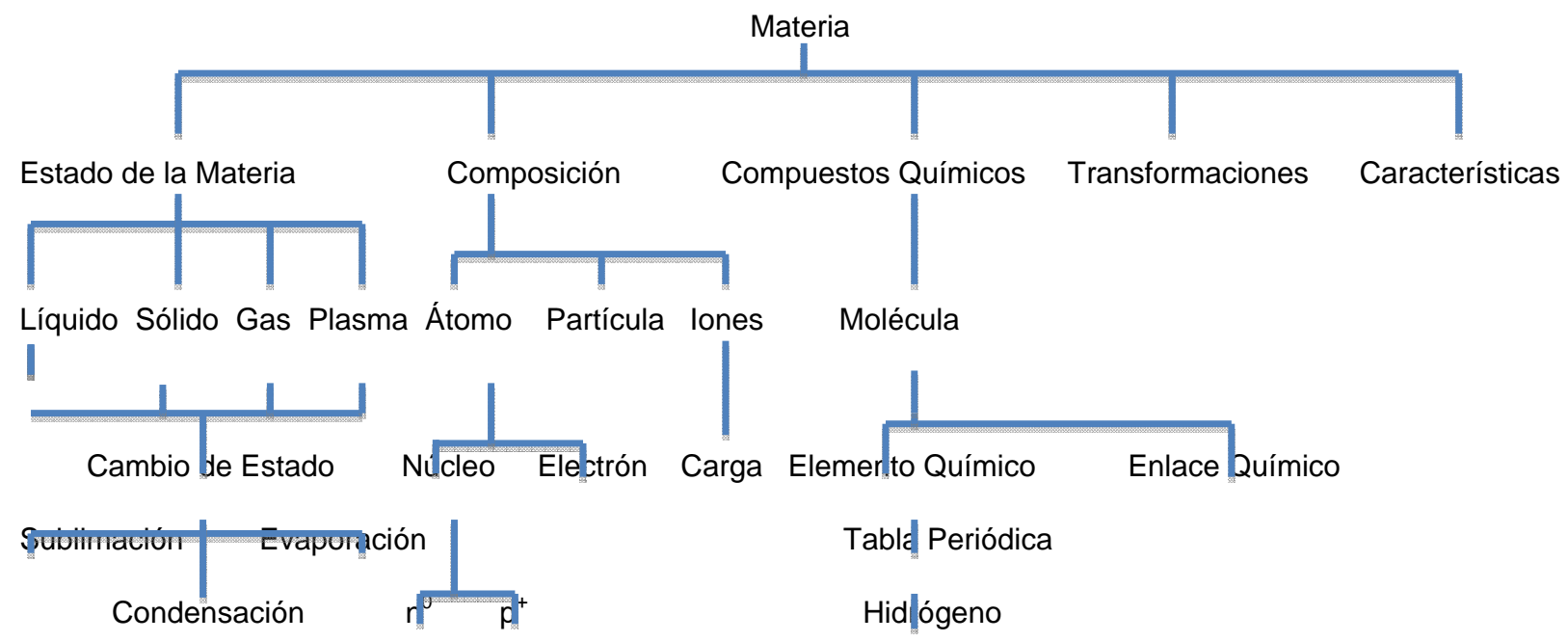

Fig. 4: Mapa de Distribución de Conceptos para $\mathrm{G}_{2}$.

$\left(G_{3}\right)$ : el mapa incluye 13 conceptos, presenta una estructura organizada que consta de cuatro tramas y cuatro niveles jerárquicos (Figura 5). La trama presenta conceptos asociados, pero los conceptos específicos están asociados solo a estados y composición de la "materia". Se observan problemas al momento de establecer relación con los conceptos de partícula, átomo y molécula. Al igual que el grupo $\mathrm{G}_{2}$ indican el concepto de plasma como un cuarto estado de la "materia", el cual si bien no es mencionado en 
los programas de estudio, refleja un grado mayor de conocimiento en relación al aspecto estados de la "materia". Establecen mejores relaciones a nivel macroscópico que microscópico.

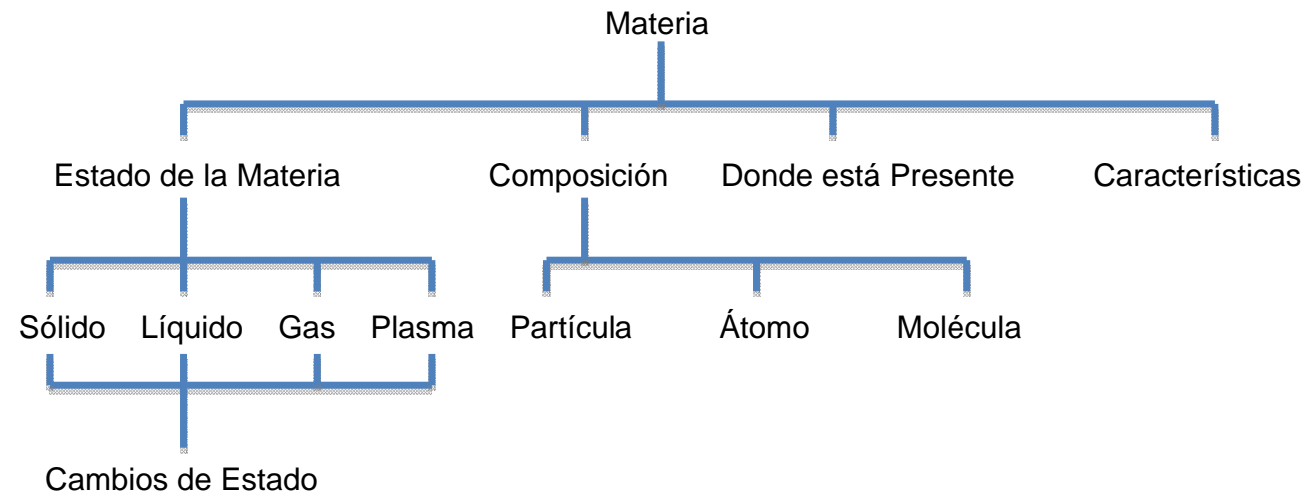

Fig. 5: Mapa de Distribución de Conceptos $\mathrm{G}_{3}$

\section{Resultados Comparativos}

Diversidad Conceptual: Ios futuros profesores son capaces de conectar al contenido la "materia" numerosos conceptos de nivel macroscópico y microscópico. Sin embargo, algunos incorporan conceptos que no están relacionados a este tema. De los aspectos que proponen en ocasiones presentan aspectos generales que no se relacionan con ningún aspecto específico $\left(G_{2}\right.$ y $\left.G_{3}\right)$ y solo un grupo de futuros profesores $\left(G_{2}\right)$ propone aspectos cuantitativos. El campo conceptual considera 7 conceptos, en los cuales $\mathrm{G}_{2}$ indica una mayoría, a diferencia de $\mathrm{G}_{1}$ y $\mathrm{G}_{3}$. Estos últimos grupos, manifiestan un campo conceptual similar y reducido. Por otra parte, respecto la diversidad de conceptos del nivel simbólico, éstos no se encuentran en ninguno de los grupos, a excepción del concepto de tabla periódica que pudiese estar vinculado a dicho nivel. No obstante, esta relación no es explicitada en los mapas de organización conceptual.

Amplitud Conceptual: aunque la cantidad de conceptos propuestos por los futuros profesores es amplia (altas frecuencias para algunos conceptos), estos valores no son representativos de una mayoría de los futuros profesores, sobre todo en los conceptos microscópicos, cuyas frecuencias son muy bajas (1). Los futuros profesores del $\mathrm{G}_{2}$ señalan mayor cantidad de conceptos microscópicos, pero con bajas frecuencias. Tanto $G_{1}$ como $G_{3}$, además de mostrar una baja diversidad, los conceptos con mayor frecuencia son los macroscópicos.

Organización Conceptual: los futuros profesores establecen relaciones directas entre cada uno de los conceptos que proponen, y en la mayoría de los casos relacionan los conceptos específicos con los conceptos generales correspondientes. Sin embargo, no existe una clara diferenciación entre lo macroscópico y microscópico, y las tramas para este último nivel son escasas. Por ejemplo, el tipo de relaciones que establece $\mathrm{G}_{2}$ tanto a nivel microscópico como macroscópico son similares, en cambio $\mathrm{G}_{1}$ y $\mathrm{G}_{3}$ establecen mejores relaciones macroscópicas que microscópicas.

\section{DISCUSIÓN E IMPLICANCIAS}

Una parte importante a considerar en la enseñanza de la química corresponde a sus niveles de representación (Martín del Pozo, 2001; Van Berkel, Pilot y Bulte, 2009). Ello implica, tratar en la enseñanza de cualquier tema los aspectos macroscópicos, microscópicos y simbólicos de forma integrada (Harrison y Treagust, 2003; Martín del Pozo, 2001). No obstante, la investigación señala la tendencia a: tratar los niveles de representación de forma separada; o a tratar solo uno de ellos, sobre todo el nivel macroscópico (Casado y Raviolo, 2005; Hilton y Nichols, 2011). Nuestros resultados, coinciden con esta tendencia, en el sentido que no observamos conceptos simbólicos en ninguno de los grupos $\left(G_{1}, G_{2}\right.$ y $\left.G_{3}\right)$ y una muy baja amplitud y diversidad en el nivel microscópico. Ello plantea una deficiencia sobre el conocimiento de los niveles de representación, más si consideramos que estos niveles son fundamentales para mejorar los resultados de nuestros estudiantes (Galagovsky, 2005, 2007; Hilton y Nichols, 2011; Reyes y Garritz, 2006; Van Berkel, Pilot y Bulte, 2009; Zaid et al., 2012). Considerar mayoritariamente aspectos macroscópicos (generales o específicos) y proponer conceptos que según los programas curriculares no forman parte del tema "materia", describe un conocimiento reducido, fragmentado y descontextualizado. 
Por otro lado, siendo importante los conceptos que se asocian para la enseñanza del tema "la materia", también importante es cómo se organizan los conceptos (Galagovsky, 2007; Martín del Pozo, 2001; Sánchez y Valcárcel, 1999). Si bien el conocimiento disciplinar que nuestros los futuros profesores es organizado, en tanto indican conceptos que van desde lo general a lo particular, se aprecia un bajo conocimiento de las relaciones entre conceptos, observándose niveles jerárquicos sin tramas estructurantes de conceptos. De hecho, todos los grupos presentan conceptos donde no elaboran tramas. Con esta baja comprensión de la estructura sustantiva y sintáctica (qué y cómo se interrelacionan los conceptos) de un tema, es muy probable que se decida seleccionar y diseñar secuencias de conceptos poco adecuadas (Arteaga e Inciarte, 2008; Garritz y Trinidad-Velasco, 2004; Hilton y Nichols, 2011).

Consideramos que estos resultados tienen implicancias en la formación inicial, dado que dan cuenta de un conocimiento disciplinar poco deseable, que no permitiría tomar decisiones adecuadas con respecto a qué y en qué orden enseñar. En otras palabras, siendo reducido el conocimiento disciplinar, es muy poco probable que el conocimiento didáctico del contenido (PCK) se desarrolle hasta llegar a un conocimiento profesional deseable (Porlán, Rivero y Martín del Pozo, 1998; Tardif, 2004; Shulman, 2005). Si lo que queremos es lograr buenos resultados en los procesos de evaluación y habilitación profesional de los futuros profesores, entonces habrá que evaluar las competencias disciplinares, pero desde una perspectiva integradora, entendiendo que para generar aprendizajes en una disciplina como la química, es necesario enseñarlas desde sus niveles de representación. En esta línea, cobrará mucho sentido, desarrollar estudios de caso, además de análisis de los planes y programas, que permitan mejorar las trayectorias formativas de los estudiantes de primaria y secundaria, como así también de los futuros profesores.

\section{CONCLUSIONES}

La cantidad de conceptos propuestos es amplia, sin embargo, no es representativa de todos los futuros profesores y tampoco de todos los niveles. De hecho, una mayoría de los conceptos indicados para enseñar "materia" corresponden al nivel macroscópico.

En esta línea, y en relación a los tipos de conceptos propuestos por el conjunto de futuros profesores, podemos concluir que la diversidad es baja. Además de ser conceptos macroscópicos tienden a ser generales, con una ausencia total del nivel simbólico. En otras palabras, el campo conceptual es reducido.

Esto concuerda con que los futuros profesores establezcan relaciones entre conceptos generales y específicos (organización). Sin embargo, las tramas conceptuales son reducidas para el nivel microscópico, más aun para el nivel simbólico y, no se aprecia una diferenciación entre los niveles. Lo cual es coherente, con que el campo conceptual sea reducido.

\section{REFERENCIAS}

Arteaga, Y. y Inciarte, A., Conocimientos que interaccionan en una clase de Ciencias Naturales. Paradigma: XXIX (1), 147-170 (2008).

Carrillo, M., Sanhueza, S. y Sánchez, A., Conocimiento que poseen los estudiantes de pedagogía en dificultades de aprendizaje de las Matemáticas (DAM). Estudios Pedagógicos: XXXV (1), 47-62 (2009).

Casado, G. y Raviolo, A., Las dificultades de los alumnos al relacionar distintos niveles de representación de una reacción química. Revista de la Facultad de Ciencias. Pontificia Universidad Javeriana: 10, 35-43 (2005)

Contreras, S., Creencias curriculares y creencias de actuación curricular de los profesores de ciencias chilenos. Revista Electrónica de Enseñanza de las Ciencias (REEC): 8 (2), 505-526 (2009).

Contreras, S., Qué piensan nuestros profesores de ciencias sobre sus clases: un acercamiento a las creencias curriculares y a las creencias de actuación curricular. Revista CIT, Formación Universitaria: 1(3), 3-11 (2008).

Chevallard, Y., La transposición didáctica: del saber sabio al saber enseñado. Aique, Buenos Aires (1991).

Galagovsky, L., La enseñanza de la química pre-universitaria: ¿Qué enseñar, cómo, cuánto, para quienes? Revista Química Viva: 4 (1), 8-22 (2005).

Galagovsky, L., Enseñar química v/s aprender química: Una ecuación que no está balanceada. Química Viva , 6 (Numero especial), 1-13 (2007). 
Garritz, A. y Trinidad-Velasco, R., El conocimiento pedagógico del contenido. Educación Química: 15 (2), 16 (2004).

Gilbert, J., y Treagust, D., Models and Modeling in Science Education. Multiple Representations in Chemical Education. Ed. Springer, UK-Australia (2009).

Gómez, M., Pozo, J. y Gutiérrez, M., Enseñando a comprender la naturaleza de la materia: el diálogo entre la química y nuestros sentidos. Educación Química: 15 (3), 198-209 (2004).

Harrison, A., y Treagust, D., The particulate nature of matter: Challenges in understanding the submicroscopic world. En J. Gilbert, O. DeJong, R. Justi, D. Chemical Education: Towards Research-based Practice, Kluwer Academic Publishers, Holland (2003).

Hilton, A. y Nichols, K., Representational Classroom Practices that Contribute to Students 'Conceptual and Representational Understanding of Chemical bonding. International Journal of Science Education: 33 (16), 2215-2246 (2011).

Martín del Pozo, R., Prospective teacher's ideas about the relationships between concepts describing the composition of matter. International Journal of Science Education: 23 (4), 353-371 (2001).

Martín del Pozo, R. y Rivero, A., Construyendo un conocimiento profesionalizado para enseñar ciencias en la Educación Secundaria: los ámbitos de investigación profesional en la formación inicial del profesorado. Revista Interuniversitaria de Formación del Profesorado: 40, 63-79 (2001).

Martínez, M. y Espinoza, A., Correlación entre mapas conceptuales y habilidades para la resolución de problemas en la unidad de equilibrio químico en la asignatura de química general. Educación Química: 20 (2), 198-207 (2009)

Moreira, M., Mapas conceptuales y aprendizaje significativo en ciencias. Revista Galáico Portuguesa de Sócio Pedagogia y Sóciolinguística: 11 (2), 143-156 (1998).

Porlán, R., Rivero, G. y Martín del Pozo, R., Conocimiento profesional y epistemológico de los profesores II: Estudios empíricos y conclusiones. Enseñanza de las Ciencias: 16 (2), 271-288 (1998).

Reyes, F. y Garritz, A., Conocimiento pedagogico del concepto de "Reacción química" en profesores univesitarios mexicanos. Revista de Investigación Educativa: 11 (31), 1175-1205 (2006).

Sánchez, G, y Valcárcel, V., Science Teachers'View and Practices in Planning for Teaching. Journal of Research in Science Teaching: 36 (4), 493-513 (1999).

Shulman, L., Conocimiento y enseñanza: Fundamentos de la Nueva reforma Profesorado. Revista de currículum y formación del profesorado: 9 (2), 1-29 (2005).

Tardif, M., Los saberes del docente y su desarrollo profesional. Narcea, Madrid (2004).

Treagust, D. y Chittleborough, T., Students'understanding of the descriptive and predictive nature of teaching models in organic chemistr. Research in Science Education: 34, 1-20 (2004).

Van Berkel, B., Pilot, A. y Bulte, M., Micro-Macro Thinking in Chemical Education: Why and How to Scape. En J. Gilbert, \& D. Treagust. Multiple Representations in Chemical Education, pp 31-54 Springer, UK (2009).

Zaid, S., Doulat, A. y Alwraikat, M., The effect of PCK on Solving Selected Chemistry Problems. International Journal of Humanities and Social Science: 2 (6), 209-212 (2012). 